\title{
Response to Letter by G. McEachen: "Receiving patients with vital signs absent from paramedics"
}

\author{
P. Richard Verbeek ${ }^{1,2}$ (D)
}

Received: 1 September 2020 / Accepted: 4 September 2020 / Published online: 4 January 2021

(c) Canadian Association of Emergency Physicians (CAEP)/ Association Canadienne de Médecine d'Urgence (ACMU) 2020

I read with interest Gord McEachen's letter [1] regarding our recent article [2]. The letter focused on paramedic safety. Dr. Nolan replied in the context of the narrower ED focus of our article [3]. I offer an additional reply.

Paramedic safety during the COVID-19 pandemic has been mitigated by approved PPE techniques and by guidance from Ontario Base Hospitals in minimizing aerosolgenerating medical procedures. Toronto Public Health has announced only two Paramedic cases of COVID-19 resulting from patient care. Each case occurred before implementing full mitigation strategies.

We appreciate the acknowledgement of the prehospital termination of resuscitation (TOR) rule developed by the Sunnybrook Centre for Prehospital Medicine [4]. Mr. McEachen describes reluctance by Base Hospital Physicians (BHP) to advise TOR. This is equated to an unnecessary safety risk due to "near misses" with high-speed crashes when running lights-and-sirens. Cardiac arrests represent a tiny proportion of lights-and-sirens transports. There were fewer cardiac arrest transports between January and June in 2020 than during similar time periods in 2019 or 2018 . Therefore, this safety risk to Paramedics is actually lower currently than previously.

The TOR rule is an "aid" and not meant to dictate a rigid "terminate or transport" approach. Transport can be warranted even though a patient meets TOR rule criteria for termination. The BHP provides a recommendation based on all available clinical information not just the TOR rule itself.

Mr. McEachen's belief that transport should occur only if there is a return of spontaneous circulation (ROSC) contradicts the TOR rule. In the Toronto region, $3.6 \%$ of patients

P. Richard Verbeek

richard.verbeek@sunnybrook.ca

1 Sunnybrook Centre for Prehospital Medicine, Sunnybrook Health Sciences Centre, Toronto, Canada

2 Division of Emergency Medicine, Department of Medicine, University of Toronto, Toronto, ON, Canada transported without a ROSC but meet one or two other components of the TOR rule for transport (i.e. have at least one shock or an EMS witnessed arrest) survive to discharge [5]. These patients would be terminated by following Mr. McEachen's advice.

Comparatively, survival was only $0.6 \%$ for patients who were transported despite meeting all three components for termination. Imposing TOR, solely because a patient does not achieve ROSC, creates an inequitable burden on the lives of some patients that Paramedics are trying to save and is ethically not justifiable.

We have recently published an out-of-hospital resuscitation/TOR protocol focusing on distributive justice. It outlines an approach that is ethically balanced and facilitates the early application of the TOR rule in clinically justifiable situations [6].

We likewise welcome open dialogue to ensure all benefits and risks are weighed appropriately as we move forward.

\section{References}

1. McEachen G. Letter to the Editor: Letter in Response to "Receiving Patients with Vital Signs Absent from paramedics. Can J Emerg Med. 2020. https://doi.org/10.1017/cem.2020.414.

2. Nolan B, et al. Recommendations for emergency departments receiving patients with vital signs absent from paramedics during COVID-19. CJEM. 2020a. https://doi.org/10.1017/cem.2020.389.

3. Nolan B. Response to Letter: "Receiving patients with vital signs absent from paramedics." Can J Emerg Med. 2020b. https://doi. org/10.1017/cem.2020.421.

4. Verbeek PR, et al. Derivation of a termination-of-resuscitation guideline for emergency medical technicians using automated external defibrillators. Acad Emerg Med. 2002;9:671-8.

5. Drennan, et al. Survival rates in OHCA patients transported without ROSC: an observational cohort study. Resuscitation. 2014;85:1488-93.

6. Leong YC, et al. Clinical considerations for out-of-hospital cardiac arrest management during COVID-19. Resusc Plus. 2020. https://doi.org/10.1016/j.resplu.2020.100027. 